\title{
Mejora continua del capital humano de instituciones educativas mixtas de básica regular, Chachapoyas, Perú, 2016
}

\section{Continuous improvement of human capital of mixed public institutions of regular basic, Chachapoyas, Perú, 2016}

\author{
Rosa Ysabel Bazán Valque ${ }^{1}$, Benjamín Roldan Polo Escobar ${ }^{2}$, Alex Javier Sánchez Pantaleón 3
}

\section{RESUMEN}

La investigación tuvo como objetivo promover la mejora continua en las instituciones educativas mixtas de Chachapoyas, aplicando el "modelo de gestión de capital humano basado en el neuroliderazgo". La población del estudio estuvo conformada por tres instituciones educativas mixtas de educación básica regular, del distrito de Chachapoyas, Perú. Compuesta por 71 docentes, siete directivos, 12 administrativos. La muestra estuvo representa por 66 ciudadanos, se utilizó el muestreo aleatorio simple. Para la recolección de datos se utilizó una encuesta antes y después de la aplicación del modelo. Se encontró que para la gestión directiva se tiene un nivel alto el $60.6 \%$ y el $10.6 \%$ tiene nivel bajo. En gestión académica tienen un nivel alto el $66.8 \%$ y el $4.4 \%$ tiene un nivel bajo. En gestión administrativa tienen un nivel alto $62.1 \%$ y el 3.3\% tiene nivel bajo. En gestión a la comunidad tienen un nivel alto $62.1 \%$ y el $4.6 \%$ tiene nivel bajo. Se concluye que el modelo permite alcanzar la mejora continua de los trabajadores de las instituciones educativas mixtas de Chachapoyas, Perú.

Palabras claves: Neuroliderazgo, mejora continua.

\begin{abstract}
The objective of the research was to promote continuous improvement in the mixed educational institutions of Chachapoyas, applying the "human capital management model based on neuro-leadership." The study population consisted of three mixed educational institutions of regular basic education, from the district of Chachapoyas, Peru. Composed of 71 teachers, seven managers, 12 administrative. The sample was represented by 66 citizens, intentional sampling was used. For data collection a survey was used before and after the application of the model. It was found that $60.6 \%$ have a high level for managerial management and $10.6 \%$ have a low level. In academic management, $66.8 \%$ have a high level and $4.4 \%$ have a low level. In administrative management $62.1 \%$ have a high level and 3.3\% have a low level. In community management, $62.1 \%$ have a high level and $4.6 \%$ have a low level. It is concluded that the model allows to achieve the continuous improvement of the workers of the mixed educational institutions of Chachapoyas, Peru.
\end{abstract}

Keywords: Neuro-leadership, continuous improvement.

\footnotetext{
Doctora en Gestión Pública y Gobernabilidad - Docente en la Facultad de Ciencias Económicas y Administrativas perteneciente a la Escuela Profesional de Administración de Empresas de la Universidad Nacional Toribio Rodríguez de Mendoza de Amazonas. Correo electrónico: rosa.bazan@untrm.edu.pe

${ }^{2}$ Doctor en Gestión Pública y Gobernabilidad - Docente en la Facultad de Ciencias Económicas y Administrativas perteneciente a la Escuela Profesional de Economía de la Universidad Nacional Toribio Rodríguez de Mendoza de Amazonas. Correo electrónico: brpoloescobar@gmail.com

${ }^{3}$ Maestro en Gestión Pública - Docente en la Facultad de Ciencias Económicas y Administrativas perteneciente a la Escuela Profesional de Economía de la Universidad Nacional Toribio Rodríguez de Mendoza de Amazonas. Correo electrónico: alex.sanchez@untrm.edu.pe
} 


\section{INTRODUCCIÓN}

La educación en la actualidad tiene como misión esencial la formación de profesionales altamente capacitados, para responder como ciudadanos responsables, competentes y comprometidos con el desarrollo social, ello implica que el proceso de formación de los docentes de cualquier nivel educativo estén llenos de conocimientos y habilidades integrales permitiendo cumplir con las funciones que requiere su profesión, por este motivo la capacitación no es una herramienta más para cumplir con las necesidades estudiantiles, sino es una obligación que debe ser practicada en cualquier institución educativa.

Para Schermerhorn (2006) el desempeño, es el balance de las personas en la institución o centro laboral. Desempeño laboral, es la calidad y cantidad de los trabajos realizados por un trabajador, es lo más importante en la productividad, contribuyendo a lograr las metas y objetivos de una organización. A lo que, Farria (1995) considera el desempeño laboral como el resultado del comportamiento de los colaboradores frente al contenido de su puesto de labores, sus actividades, tareas y atribuciones depende de un proceso de mediación entre él y la institución.

Según Cuentas (2018) y Davenport (2006), plantean a la gestión del capital humano como un factor importante para la mejora de la educación. Ambos estudios reconocen que una eficiente gestión del capital humano conlleva el desarrollo de las siguientes estrategias: 1. Contratación de personas adecuadas. 2. Un buen clima institucional, 3 . Constitución del capital humano y 4. Retención del capital humano.

La gestión del capital humano apunta a configurarse como el proceso administrativo de inserción del componente humano, en función de las competencias individuales de cada persona. Según Majad (2016), indica que la realización de los objetivos organizacionales, debe de respetar las etapas de planificación, ejecución y control, bajo los principios de identidad, cultura y filosofía colectiva. En este punto, las organizaciones educativas no están separadas de esta categorización, al contrario, se encuentran determinadas por ella.

Una institución educativa que comprenda y le interese implantar un sistema educativo orientado al logro de la calidad de la educación, según Martín, Segredo, \& Perdomo (2013), deben transformarse en un agente de cambio que establezca estándares y pautas para los procesos de enseñanza aprendizaje, poseer los recursos para afrontar los costos y adquirir tecnología de punta, crear la infraestructura necesaria, mantenerla y actualizarla constantemente, así como diseñar una estructura organizacional con esquemas administrativos flexibles e innovadores que den respuesta a las necesidades sociales.

La gestión es concebida por , como algo que no está aislado a un compartimiento hermético dentro de la organización, sin contacto con las demás actividades, sino que traspasa todos los ámbitos y niveles del actuar de la organización, que involucra y está presente en todos. En la gestión se puede observar y diferenciar campos de acción, interrelacionados, entre los cuales están la gestión de producción, de talento humano, financieros, de mercadeo, sociales y políticas, legales y tributarias, ambientales, entre otras.

Según Majad (2016), menciona a (Galleto, 2003, p. 80) que la planificación es fundamental, y se refiere al componente del proceso administrativo de la empresa que tiene por objetivo la selección de las actividades a realizar y fijación de los objetivos de la organización.

En el ámbito organizacional educativo, Cuesta (2002), desarrollar un conjunto de mecanismos informativos, de ejecución y control, que puede utilizar la dirección, para aumentar la probabilidad que el comportamiento de las personas que forman parte de la institución sea coherente con sus objetivos. Esta coordinación e integración del talento humano se puede explicar a través de la gestión por competencias Los enfoques de competencias comparten puntos comunes, como el hecho de que cada competencia presenta un nombre en cuanto definición verbal precisa: identificación, autoconfianza, información, orientación, flexibilidad, liderazgo; significando que cada competencia tiene niveles que reflejan conductas observables.

Asimismo Montilva (2002), indica que en el campo de la docencia, la gerencia educativa es considerada como un proceso de coordinación de una institución por medio del ejercicio de habilidades directivas encaminadas a planificar, organizar, coordinar y evaluar. El Director de la institución educativa ahora es llamado gerente educativo, se vale del proceso de planificación, el cual refiere definir metas y estrategias gerenciales para alcanzarlas. El perfil del directivo comprende las competencias gerenciales: liderazgo, capacidad de dirección y gestión del cambio.

En ese sentido Núñez \& Días (2017), coincide con (Arbaiza, 2011), que los modelos sobre las competencias gerenciales en el contexto latinoamericano, consiste en un pentágono conformado por las siguientes competencias: manejo personal, manejo de acción, competencias de manejo de entorno, manejo interpersonal y manejo de la influencia.

Es necesario recalcar que el proceso educativo, es también de carácter social, sustentado por Apodaca, Ortega, Verdugo, \& Reyes (2017), en el análisis nos dimos cuenta de la evolución que se ha dado a través del tiempo, considerando importante la formación de recursos humano como resultado a la atención de las necesidades de la sociedad.

Según Angulo, Quejado, \& Yáñez (2012), coincide 
con, , que la relación existente entre ingresoseducación en México, fue obtenido a través del cálculo de las tasas de rentabilidad de la educación, por lo tanto la teoría del capital humano sigue siendo la base para la explicación de la determinación de los salarios y sus diferencias.

La mejora continua, según, Kaizen (1986). Implica, tanto la implantación de un sistema como el aprendizaje continuo de la organización, el seguimiento de una filosofía de gestión, y la participación activa de todas las personas. Con la mejora continua si es posible influir en el deseo de cambio en la mentalidad del personal, ayudando a lograr el éxito esperado" (Cárdenas, 2003, p. 50).

En las Instituciones Educativas Públicas, la gestión actual se desarrolla en un contexto de incertidumbre, conflictos y riesgos, es una deficiencia, en comparación con lo que el neuroliderazgo introduce como nueva forma de pensar, decidir y hacer en la gestión de recursos humanos. (Córdova \& Molina, 2015,p.1)

Vallejo, et al. (2017) sostiene que resulta tan difícil razonar con líderes que están emocionalmente desmotivado; no importa la sensatez del argumento que se le ofrezca desde un punto de vista lógico: este no tiene ninguna validez si no es acorde con la convicción emocional del momento. Los sentimientos son autos justificadores y cuentan con un conjunto de percepciones y "pruebas" propios, además se argumenta los desconocimientos de neuroliderazgo lo que dificulta en la toma de decisiones de las empresas.

El presente trabajo de investigación se enfocó en el capital humano y la mejora continua de la educación, para lo cual se planteó como objetivo general promover la mejora continua en las instituciones educativas mixtas de Chachapoyas, aplicando el "modelo de gestión de capital humano basado en el neuroliderazgo.

\section{MATERIAL Y MÉTODO}

Diseño: La investigación estuvo enmarcado en el enfoque cuantitativo de tipo propositivo en tanto que se elaboró un modelo, con diseño de campo y modalidad de proyecto factible, no experimental de tipo transeccional.

Población (N). 07 Directivos, 71 docentes, 12 administrativos de las instituciones educativas mixtas de Chachapoyas.

Relación de Instituciones Educativas y número de directivos, docentes y administrativos.

\begin{tabular}{|c|l|c|c|c|}
\hline \multirow{4}{*}{$\begin{array}{c}\text { NSTITUCIONES } \\
\text { EDUCATNAS }\end{array}$} & \multicolumn{1}{|c|}{ CARGOS } & $\begin{array}{c}\text { NÚMERO } \\
\text { DE } \\
\text { MUJERES }\end{array}$ & $\begin{array}{c}\text { NÚMERO } \\
\text { DE } \\
\text { VARONES }\end{array}$ & TOTAL \\
\hline \multirow{4}{*}{\begin{tabular}{c} 
Miguel Rubio" \\
\cline { 2 - 5 }
\end{tabular}} & Directivos & 0 & 2 & 2 \\
\cline { 2 - 5 } $\begin{array}{c}\text { "Santiago Antúnez } \\
\text { de Mayolo" }\end{array}$ & Docentes & 16 & 9 & 25 \\
\cline { 2 - 5 } & Administrativos & 2 & 1 & 3 \\
\cline { 2 - 5 } & Docentes & 14 & 7 & 21 \\
\hline \multirow{3}{*}{$\begin{array}{c}\text { "San Juan de la } \\
\text { Libertad" }\end{array}$} & Administrativos & 2 & 1 & 3 \\
\cline { 2 - 5 } & Docentes & 20 & 5 & 25 \\
\cline { 2 - 5 } & Administrativos & 1 & 5 & 6 \\
\hline
\end{tabular}

\section{Muestra (n) y Muestreo:}

La muestra estuvo constituido por 66 ciudadanos, elegidos por muestreo aleatorio simple. El tamaño de la muestra se obtuvo aplicando el método aleatorio simple, utilizando la siguiente formula:

$$
\mathrm{n}=\frac{Z^{2} * P * Q * N}{(N-1) e^{2}+Z^{2} * P * Q}
$$

Dónde:

$\mathrm{Z}=$ nivel de confianza.

$\mathrm{P}=$ probabilidad de éxito $50 \%$

$\mathrm{Q}=$ probabilidad de fracaso $50 \%$

$\mathrm{N}=$ universo

$\mathrm{e}=$ Error de estimación admitido.

$\mathrm{n}=$ Tamaño de la muestra.

\section{Desarrollando:}

$$
\begin{aligned}
& \mathrm{n}=\frac{(1.96)^{2} * 0.50 * 0.50 * 17924}{(17924-1)(0.12)^{2}+(1.96)^{2} * 0.50 * 0.50} \\
& \mathrm{n}=66 \text { Ciudadanos }
\end{aligned}
$$

\section{Construcción del instrumento de mejora continua:}

Se construyó un modelo de gestión de capital humano basado en el neuroliderazgo y sus objetivos específicos, a) diagnosticar la situación actual de las instituciones educativas de la provincia de Chachapoyas, región Amazonas, con relación a la gestión del capital humano b) Aplicación del modelo de gestión del capital humano para el fortalecimiento del trabajo educativo en las instituciones educativas públicas de la provincia de Chachapoyas, región Amazonas. c) evaluar la mejora continua con la aplicación del modelo de gestión del capital humano basado en el neuroliderazgo.

El modelo de gestión de capital humano basado en el neuroliderazgo consistió en: 1. Desarrollo de capacidades: taller de autoestima, taller de liderazgo y reuniones de integración. 2. Trabajo de gestión: trabajo colegiado, delegación de funciones cooperativas y generar identidad institucional. El modelo fue validado a criterio de expertos.

Antes y después de la aplicación del modelo de 
gestión de capital humano basado en el neuroliderazgo se aplicó una encuesta de 16 preguntas, la misma que fue sometida a juicio de expertos y confiabilidad según coeficiente Alfa de Cronbach (0.73). Para el análisis de datos se utilizó el software estadístico SPSS versión 2

\section{RESULTADOS}

Tabla 1. Porcentaje de nivel de gestión directivo en pre encuesta y post encuesta.

\begin{tabular}{ccc}
\hline $\begin{array}{c}\text { Nivel de } \\
\text { gestión }\end{array}$ & $\begin{array}{c}\text { Porcentaje } \\
\text { pre test }\end{array}$ & $\begin{array}{c}\text { Porcentaje } \\
\text { post test }\end{array}$ \\
\hline Alto & $35.2 \%$ & $60.6 \%$ \\
Regular & $19.6 \%$ & $28.8 \%$ \\
Bajo & $45.2 \%$ & $10.6 \%$ \\
\hline Total & $100 \%$ & $100 \%$ \\
\hline
\end{tabular}

Tabla 2. Porcentaje de nivel de gestión académica en pre encuesta y post encuesta.

\begin{tabular}{ccc}
\hline $\begin{array}{c}\text { Nivel de } \\
\text { Gestión }\end{array}$ & $\begin{array}{c}\text { Porcentaje } \\
\text { pre test }\end{array}$ & $\begin{array}{c}\text { Porcentaje } \\
\text { post test }\end{array}$ \\
\hline Alto & $23.5 \%$ & $66.8 \%$ \\
Regular & $48.9 \%$ & $28.8 \%$ \\
Bajo & $27.6 \%$ & $4.4 \%$ \\
\hline Total & $100 \%$ & $100 \%$ \\
\hline
\end{tabular}

Tabla 3. Porcentaje de nivel de gestión administrativa en pre encuesta y post encuesta.

\begin{tabular}{ccc}
\hline $\begin{array}{c}\text { Nivel de } \\
\text { Gestión }\end{array}$ & $\begin{array}{c}\text { Porcentaje } \\
\text { pre test }\end{array}$ & $\begin{array}{c}\text { Porcentaje } \\
\text { post test }\end{array}$ \\
\hline Alto & $41.2 \%$ & $62.1 \%$ \\
Regular & $13.1 \%$ & $19.6 \%$ \\
Bajo & $45.7 \%$ & $3.3 \%$ \\
\hline Total & $100 \%$ & $100 \%$ \\
\hline
\end{tabular}

Tabla 4. Porcentaje de nivel de gestión de la comunidad en pre encuesta y post encuesta.

\begin{tabular}{ccc}
\hline $\begin{array}{c}\text { Nivel de } \\
\text { Gestión de la }\end{array}$ & $\begin{array}{c}\text { Porcentaje } \\
\text { pre test }\end{array}$ & $\begin{array}{c}\text { Porcentaje } \\
\text { post test }\end{array}$ \\
\hline Alto & $22.9 \%$ & $62.1 \%$ \\
Regular & $38.4 \%$ & $33.3 \%$ \\
Bajo & $38.7 \%$ & $4.6 \%$ \\
\hline Total & $100 \%$ & $100 \%$ \\
\hline
\end{tabular}

\section{DISCUSIÓN}

Teniendo en cuenta los datos de la (tabla 1), se puede observar que la mayoría de encuestados (45.2\%) indicaron que tienen una gestión directiva baja frente al tema de mejora continua y un (19.6\%) respondió que tiene una posición regular frente al tema. Significando que existe un problema y que debe corregirse para alcanzar la mejora continua, por lo que después de aplicarse el modelo de gestión del capital humano basado en el neuroliderazgo, la mayoría de encuestados (60.6\%) indicaron que tienen una gestión directiva alta frente al tema de mejora continua, y que solo el $(10.6 \%)$, respondió que tienen una posición baja frente al tema. Demostrando que se si se alcanzó lo esperado.

Teniendo en cuenta los datos de la (tabla 2), se puede observar que la mayoría de encuestados (48.9\%) indicaron que tienen una gestión académica regular frente al tema de mejora continua y un $(23.5 \%)$ respondió que tiene una posición alta frente al tema. Significando que existe un problema y que debe corregirse para alcanzar la mejora continua, por lo que después de aplicarse el modelo de gestión del capital humano basado en el neuroliderazgo, la mayoría de encuestados (66.8\%) indicaron que tienen una gestión académica alta frente al tema de mejora continua, y que solo el $(4.4 \%)$, respondió que tienen una posición baja frente al tema. Demostrando que se si se alcanzó lo esperado.

Teniendo en cuenta los datos de la (tabla 3), se puede observar que la mayoría de encuestados (45.7\%) indicaron que tienen una gestión administrativa baja frente al tema de mejora continua y un $(13.1 \%)$ respondió que tiene una posición regular frente al tema. Significando que existe un problema y que debe corregirse para alcanzar la mejora continua, por lo que después de aplicarse el modelo de gestión del capital humano basado en el neuroliderazgo, la mayoría de encuestados (62.1\%) indicaron que tienen una gestión administrativa alta frente al tema de mejora continua, y que solo el $(3.3 \%)$, respondió que tienen una posición baja frente al tema. Demostrando que se si se alcanzó lo esperado.

Teniendo en cuenta los datos de la (tabla 4), se puede observar que la mayoría de encuestados (38.7\%) indicaron que tienen una gestión de la comunidad baja frente al tema de mejora continua y un (22.9\%) respondió que tiene una posición regular frente al tema. Significando que existe un problema y que debe corregirse para alcanzar la mejora continua, por lo que después de aplicarse el modelo de gestión del capital humano basado en el neuroliderazgo, la mayoría de encuestados (62.1\%) indicaron que tienen una gestión de la comunidad alta frente al tema de mejora continua, y que solo el $(4.6 \%)$, respondió que tienen una posición baja frente al tema. Demostrando 
que se si se alcanzó lo esperado.

Los resultados obtenidos en post test de la investigación no coinciden con los realizados por , al utilizar un enfoque mixto, desde el punto de vista cuantitativo realizó un análisis estadístico de las encuestas y cualitativo realizo una descripción detallada de experiencias, actitudes pensamientos y reflexiones, con una población compuesta por los directivos y docentes de la especialidad de Lengua y Literatura, con más de diez años de servicio en la Facultad de Educación de una universidad privada, con una muestra no probabilística intencional, aplicando como técnica la entrevista y la encuesta.

Como resultado de la interacción, Directivos, Docentes e información obtenida de los instrumentos utilizados, como es la encuesta y la entrevista, se tiene resultados bajo 04 categorías consideradas por (Cuentas, 2018). 1. Contratación de inversores de capital humano: El $71 \%$ de los docentes expresó desconocerlo. Directivos y docentes aseguraron que el Decano es la persona que lo realiza y controla toda la información. 2. Disposición del clima institucional: el $71 \%$ de los docentes encuestados manifestó que las reuniones entre directivos y docentes son esporádicas, que a pesar que sus aportes y opiniones no son tomados en cuenta, gozan de total autonomía para el desempeño de su cátedra. 3. Constitución del capital humano: 57\% manifestaron que son pocas las veces que se desarrollan. Es decir, se realizan, pero no como parte de un proceso de planificación sobre la base de las demandas de conocimientos de los docentes, sino a criterio de los directivos y/o alguna demanda urgente que se presente. 4. Retención del capital humano: $71 \%$ expresó no conocer algún incentivo que otorgue la Institución, a pesar que los directivos expresan la existencia de incentivos, considerando q el único incentivo conocido por algunos docentes y aceptado por los directivos es el reconocimiento personal y moral a docentes que han sobresalido en su cátedra.

Según resultados de pre test, coincido con lo obtenido por Majad ( 2016), con los resultados de su investigación "Gestión del talento humano en las organizaciones educativas en su Dimensión Gestión, que considera como indicador 1. Planificación: referente a la fijación de los objetivos de la institución, tanto a largo como a corto plazo, un $65,9 \%$ señaló que se hace a veces. 2 . Toma de decisiones: un $63 \%$ del estrato docente respondió a veces. 3. Ejecución: el 55,8\% del estrato docente señaló que los directivos nunca ejercen la responsabilidad de dirigir las actividades de los subordinados para lograr objetivos organizacionales, mientras que el $62,5 \%$ del estrato directivo respondió hacerlo siempre. 4. Control: un 6,7\% del estrato docente respondió nunca. 5. Responsabilidad compartida: existe una situación medianamente favorable, ya que los objetivos de trabajo están amparados en planeamientos comunes y con responsabilidad mutua compartida, además, en líneas generales, el directivo de la institución es la cabeza visible de la organización e interactúa con personas dentro y fuera de ella.

\section{CONCLUSIONES}

1. El nivel de gestión directivo es alto en un $60 \%$ y el $10.6 \%$ tiene un nivel bajo.

2. El nivel de gestión académica es alto en un $66.8 \%$ y el $4.4 \%$ tiene un nivel bajo.

3. El nivel de gestión administrativa es alto en un $62.1 \%$ y el $3.3 \%$ tiene un nivel bajo.

4. El nivel de gestión de la comunidad es alto en un $62.1 \%$ y el $4.6 \%$ tiene un nivel bajo.

\section{REFERENCIAS BIBLIOGRÁFICAS}

Angulo, G. M., Quejado, R., \& Yáñez, M. (2012). Educación, mercado de trabajo y satisfacción laboral: el problema de las teorías del capital humano y señalización de mercado. Scielo-Revista de la Educación

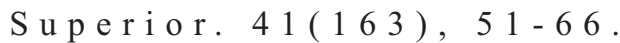
http://www.scielo.org.mx/scielo.php?script $=\mathrm{s} \mathrm{c} \mathrm{i} \mathrm{art} \mathrm{text \& pid=S} 0185$ $276020 \overline{1} 2000300002 \& \operatorname{lng}=$ es\&tlng $=$ es

Apodaca, G. U., Ortega, L. P., Verdugo, 1. E., \& Reyes, L. E. (2017). Modelos Educativos para la educación en Salud. Ra Ximhai, 13 ( 2 ), $77-86$. https://www.redalyc.org/articulo.oa?id=461 $/ 46154510006$

Arana, A. (2012). Neuroliderazgo: Una perspectiva innovadora del liderazgo. Recuperado el 26 $d e \quad 8 \quad d e \quad 2016, \quad \mathrm{de}$ file://C:/Users/ACER/Downloads/Articulo _Neuroliderazgo_Una_Perspectiva.pdf

Barceinas, F., \& Raymond Bara, J. L. (2001). Capital humano y rendimientos de la educación en México. Universitat Autònoma de Barcelona. https://ddd.uab.cat/record/38375

Braidot, N. (s.f.). Las neurociencias como fuente de oportunidades para la innovación en las organizaciones. Braidot Center. Obtenido de file://C:/Users/ACER/Downloads/Neurom anagement_neuroliderazgo_neuromark.pdf

Córdova, M., \& Molina, C. (14 de mayo de 2015). Un neuroliderazgo para la satisfacción laboral 
en las instituciones educativas. Gerencia y calidad educativa.

Cuentas, S. (2018). La Gestión del Capital Humano en la Unidad Académica de Educación superior: analysis of a university. E d u c a c i ón, 27 ( 53 ) , 57 - 72 . http://www.scielo.org.pe/scielo.php?pid=S $\begin{array}{llll}1 & 0 & 1 & 9\end{array}$ 94032018000200004\&script=sci_arttext

Cuesta, A. (2002). Gestión del Comportamiento. Analisis y Proyección de los Recursos Humanos. La Habana: Academia.

Davenport, T. (2006). Capital humano. Barcelona: Gestión 2000.

Esquivel, A., Castellanos, G. M., \& León, R. (2017). Mejora Continua de los procesos de gestión del conocimiento en instituciones educativas superior ecuatorianas. Retos de la Dirección, 11(2), 56-72. Obtenido de http://scielo.sld.cu/pdf/rdir/v11n2/rdir0521 7.pdf

Faria, F. (1995). Desarrollo Organizacional. Enfoque Integral. Noriega Editores. México.

Kaizen, M. (1986). Mejora continua. Principios [en línea]. Recuperado el 6 de julio de 2016, de http://www.competitividup.com/?p=535

Majad, M. A. (2016). Gestión del talento humano en organizaciones educativas. Revista de investigación, 40(88), $148-165$. https://www.redalyc.org/pdf/3761/3761471 31008.pdf

Martín, X., Segredo, A. M., \& Perdomo, I. (2013). Capital humano, gestión académica y desarrollo organizacional. Educación Médica Superior, 27(3), 288-295. http://scielo.sld.cu/scielo.php?pid=s0864$21412013000300014 \&$ script $=$ sci_arttext\&t lng $=$ en

Montilva, M. (2002). Educación y Gerencia: Perfiles Competitivos. México UNAM.

Nuñez, N., \& Dias, D. (2017). Perfil por competencias gerenciales en directivos de instituciones educativas. Estudios pedagógicos (Valdivia), 43(2), 237-252. https://dx.doi.org/10.4067/S 0718 07052017000200013

Simón, H. (2002). El Comportamiento Administrativo. México.
Schermerhorn, J. (2006). Administración. México: Limusa Wiley, Descripción Física.

Vallejo, D; Abarca C; Uquillas G. y Ramírez R. (2017). Inteligencia emocional y el neuroliderazgo en las Empresas pública, Revista Observatorio de la Economía Latinoamericana, Ecuador. 